De Miguel Díaz, Mario (2015). Ideología y pedagogía empírica: cuestiones para un debate. Revista de Investigación Educativa, 33(2), 269-287.

DOI: http://dx.doi.org/10.6018/rie.33.2. 226611

\title{
Ideología y pedagogía empírica: cuestiones para un debate
}

\section{Ideology and Empirical Pedagogy: Questions for debate}

\author{
Mario de Miguel Díaz \\ mario@uniovi.es
}

\begin{abstract}
Resumen
Los investigadores empíricos en educación también tenemos la obligación de "hacer pedagogía" explicando de manera comprensible lo que hacemos, como lo hacemos y, sobre todo, para qué. Durante los últimos años constatamos la tendencia a sustituir la lógica propia de la investigación educativa-conocer/decidir/mejorar-por otra orientada primordialmente hacia la publicación de trabajos en revistas con reconocidos índices de impacto. Con este estudio pretendemos efectuar una reflexión sobre algunas de las debilidades conceptuales y metodológicas que presentan muchos de los trabajos empíricos realizados en el campo de la educación que pueden generar resultados poco creíbles e interpretaciones sesgadas de los mismos. En definitiva, efectuar una llamada de atención para que la ideología e intereses académicos de los investigadores no prevalezcan sobre los fundamentos epistemológicos, metodológicos y éticos que deben regir todo trabajo sobre pedagogía empírica.

Palabras clave: revisión crítica de estudios empíricos, problemas teóricos y metodológicos, responsabilidad ética del investigador, ideología e investigación empírica.
\end{abstract}

\begin{abstract}
As empirical researchers in education, we must also use pedagogy in order to explain what we do and how we do it, in a clear and understandable manner, and most importantly, why we do it. In recent years we verified the tendency to replace the logic of educational research know / decide / improvement - with another one primarily oriented toward publishing papers
\end{abstract}

Correspondencia: Mario de Miguel Díaz. mario@uniovi.es 
in high impact journals. With this study we aim to make a reflection on some of the conceptual and methodological weaknesses many of the empirical studies in the field of education have, that can generate implausible results and biased interpretations. Ultimately, it is about making a wake-up call to researchers, so that their ideology and academic interest do not prevail over the epistemological, methodological and ethical principles that should govern all empirical work on pedagogy.

Keywords: critical review of empirical studies, theoretical and methodological problems, ethical accountability of researcher, ideology and empirical research.

\section{Introducción}

Durante los últimos años es frecuente escuchar en los medios políticos y de difusión que hay que "hacer pedagogía" con el fin de que la sociedad llegue a asimilar determinados supuestos ideológicos y normativas que se intentan puntualmente establecer o difundir. En estos casos se utiliza el término "pedagogía" como sinónimo del concepto de "didáctica" en tanto que alude al "procedimiento o método para hacer comprensible cuestiones complejas que requieren un análisis e interpretación correcta". Sea cual sea la finalidad con que se utiliza esta expresión, y a pesar de su limitada acepción, es meritorio este reconocimiento público sobre la utilidad social que esta disciplina puede aportar al interés común.

Por otro lado, es igualmente habitual observar que se utilizan los resultados de algunas investigaciones educativas como "argumentos" para descalificar determinadas decisiones políticas o justificar nuevas normativas u opciones ideológicas que se pretenden establecer. Observamos que, con frecuencia, se utilizan datos empíricos relacionados con las investigaciones educativas de forma parcial, descontextualizada y sin tener en cuenta los requisitos y condicionantes que necesariamente siempre se deben tener presentes a la hora de efectuar su interpretación y utilización. Esto es lo que está sucediendo con los estudios de PISA. Habitualmente se efectúa una "lectura sesgada" de los datos que aportan estos Informes destacando aquellos -tasas de fracaso, porcentaje de abandonos, rankings entre comunidades, etc.- que en cada momento sirven para justificar los intereses de un determinado grupo o partido y descalificar al contrario.

Esta forma partidista y poco crítica en la utilización de los datos que aportan los informes de investigación es la que queremos denunciar ya que, desde nuestro punto de vista, no es lo mismo "hacer pedagogía" que "utilizar a la pedagogía". Como miembros de un colectivo que, en el conjunto de las disciplinas académicas, tiene la competencia de la investigación empírica de los fenómenos educativos no podemos permanecer al margen de esta utilización sesgada de los datos obtenidos con nuestros trabajos. Quienes nos dedicamos a la pedagogía empírica de alguna forma debemos sentirnos interpelados ante esta "manipulación" interesada con fines espureos.

Los datos que aportan los trabajos, en principio, pueden ser tipificados como "neutros" -siempre que no haya habido sesgos en el proceso de su elaboración- y su "significado" depende de los criterios que se utilizan para su interpretación. Por ejemplo, el número de asistentes a un acto puede valorarse como escaso o numeroso en función de diversas variables que pueden incidir sobre el mismo. Dependiendo de aquellas que se consideren en cada caso el resultado de la valoración será claramente distinto. 
El significado que se le atribuye a un dato en un momento y contexto determinado depende, por tanto, del criterio específico que utiliza quien efectúa su interpretación, criterio que puede o no ser representativo respecto al conjunto de parámetros teóricos y metodológicos que han sido tenidos en cuenta por el investigador para su obtención.

Este simple hecho nos advierte a todos los que nos dedicamos a la investigación educativa de un riesgo previsible-suministrar datos sobre los fenómenos educativos y dejar que otros establezcan los criterios para su interpretación- que es preciso tener en cuenta e intentar remediar en la medida de lo posible. La forma de presentar y difundir los resultados de nuestros estudios puede dar pie a que se puedan utilizar de forma sesgada y partidista. Si realmente esto es lo que está sucediendo, debemos reflexionar como colectivo dedicado a la investigación educativa si tenemos alguna responsabilidad sobre ello y preguntarnos porqué sucede y cómo podemos actuar para promover criterios de racionalidad en la interpretación de los resultados que otros realizan de nuestros trabajos. De alguna forma también nosotros tenemos que "hacer pedagogía" y explicar lo que hacemos, cómo lo hacemos y sobre todo, para qué.

Aunque es difícil controlar los "criterios partidistas" que otros pueden utilizar a la hora de interpretar los datos de nuestros estudios, podemos y debemos controlar aquellos que tienen su origen en las debilidades conceptuales subyacentes en nuestra forma de realizar trabajos empíricos, es decir, en la carencia o no de explicitación de la fundamentación epistemológica, metodológica y ética que condiciona nuestra praxis. En la medida que nuestras investigaciones no están justificadas teóricamente, damos más opción a que sea cuestionada su credibilidad y, en consecuencia, los resultados que aportan. De ahí el objetivo que orienta el presente artículo: reiniciar un debate sobre algunas de las debilidades conceptuales y metodológicas que presentan muchos de los trabajos empíricos realizados en el campo de la educación que pueden generar resultados poco creíbles $e$ interpretaciones sesgadas de los mismos. En definitiva, efectuar una aproximación a las relaciones entre ideología y pedagogía empírica.

\section{Ideología e investigación educativa}

Aunque existen diversas concepciones y aproximaciones al concepto "ideología" (Lenk 1974, Trías 1975, Rossi 1980, Popkewitz 1988), quienes nos situamos en el plano empírico habitualmente utilizamos este término para designar al conjunto de supuestos o ideas fundamentales necesarias para justificar toda praxis humana. Se trataría, pues, de una forma de pensamiento práctico vinculado a la acción. Al igual que otros profesionales, los investigadores también dirigen su praxis partiendo de determinados supuestos o concepciones teóricas sobre cómo se debe investigar y qué papel juegan los resultados de la investigación en la transformación de la realidad social y educativa. De ahí que entendamos como "ideología del investigador" a la "cosmovisión" que tienen quienes se dedican a esta tarea y que se manifiesta en su manera de ver e interpretar la naturaleza, finalidad y metodología propias de la investigación educativa.

Interesa resaltar esta aproximación al concepto de ideología como cosmovisión para constatar que no existe quehacer humano que pueda analizarse al margen de las concepciones y supuestos teóricos que subyacen a todo tipo de pensamiento práctico y, en consecuencia, a la acción investigadora. Así pues, ciencia e ideología son térmi- 
nos interrelacionados aunque algunos autores tratan de contraponerlos -defendiendo la necesidad de hacer ciencia para superar la ideología- mientras que otros llegan a identificarlos postulando que la ciencia resulta de factores no científicos, hasta el punto que es una ideología más (Feyerabend, 1982). Sin llegar a afirmaciones tan extremas consideramos muy importante que en cada trabajo se especifique adecuadamente las bases conceptuales y metodológicas que determinan tanto las decisiones y procesos prácticos relativos a la investigación realizada como a los resultados.

Partiendo de este supuesto, un sencillo ejercicio de metaevaluación nos permite afirmar que es común en la mayoría de los trabajos empíricos la carencia de bases teóricas sólidas que permitan estimar la "cosmovisión" que el investigador utiliza como marco de referencia de su trabajo. Desde nuestro punto de vista constituye una debilidad acusada que tiene la investigación empírica en educación. Por ello, dado que la ciencia se desarrolla resolviendo incertidumbres, constituye un grave error metodológico que una comunidad de científicos ignore o margine los problemas que más cuestionan la credibilidad y utilidad de sus trabajos (Lakatos, 1982). Antes de fijarse en los sesgos que "los otros" efectúan al leer e interpretar los "resultados" de nuestros trabajos empíricos deberemos analizar las limitaciones conceptuales que presentan los mismos; es decir, en la ideología que determina nuestra praxis investigadora.

Este es el enfoque que pretendemos abordar en este trabajo. Tratamos de efectuar una reflexión sobre algunos aspectos del proceso investigador que consideramos deben ser justificados detalladamente ya que están mediatizados por la ideología personal del investigador, a saber: su concepto sobre la naturaleza de la investigación empírica, la selección que efectúa de los problemas objeto de la investigación, la elección del paradigma metodológico utilizado para realizar la investigación, el tipo de soporte o fundamento sobre el que justifica la racionalidad científica del trabajo, los procedimientos que el autor utiliza para la difusión de los resultados y, finalmente, el compromiso ético que manifiesta con la tarea que realiza. Somos conscientes de que la ideología del investigador está presente en todo análisis de la realidad educativa (Castané, 1986) y en todas las fases del proceso investigador por lo que la concreción de nuestro análisis a estos seis aspectos puede ser juzgada como parcial. Nuestro propósito se limita a reabrir un camino de revisión crítica -ya planteado hace tiempo por otros colegas (Dendaluce, 1995, 1998, 1999; Bartolomé, 2000)- esperando se considere oportuno y sea objeto de debate.

\section{Epistemología y pedagogía empírica}

En el ámbito de las disciplinas humanas y sociales identificar el concepto de "conocimiento científico" nunca ha sido un asunto sencillo ya que previamente el sujeto se debería decantar por un modelo de ciencia basado en la "explicación causal" siguiendo la tradición galileana frente a otro de inspiración aristotélica que se orienta a "dar razón a los hechos" (explicación teleológica). Lógicamente los enfoques y planteamientos que conllevan ambas tradiciones científicas son muy distintos y han generado constantes polémicas -la mayoría aún no resueltas- que han determinado que la epistemología de las ciencias humanas y sociales haya constituido una de las cuestiones filosóficas más controvertidas durante las últimas décadas. El debate 
sobre si son realmente ciencias y el tipo de métodos propios que requieren siempre ha constituido un tema recurrente sujeto a interpretaciones diversas.

No obstante, aportaciones relativamente recientes -especialmente la de Hesse (1980) - plantean que los problemas epistemológicos de las ciencias de la naturaleza como de las llamadas ciencias humanas y sociales son muy semejantes ya que ni los datos pueden ser separados de la teoría ni los hechos pueden ser reconstruidos al margen de su interpretación De ahí que, aunque la explicación galileana de la ciencia haya sido considerada habitualmente como mas solvente que la aristotélica, durante los últimos años la prevalencia epistemológica de las llamadas ciencias de la naturaleza no se sostiene ni desde el punto de vista teórico ni metodológico ya que también este tipo de epistemología tiene sus propios problemas y limitaciones (Mardones, 1991).

De ahí que, frente a una teoría dualista de la explicación científica, se haya ido abriendo paso una nueva epistemología que no se limita a cuestiones propias de la lógica formal sino que pone énfasis sobre una concepción distinta del hombre y la sociedad, y que está preocupada por resaltar "las peculiaridades del objeto de la investigación". Ello no sólo ha posibilitado el rechazo de los exclusivismos ideológicos sobre el concepto de ciencia sino también abrir pasos hacia otras teorías que permiten abordar las cuestiones epistemológicas y metodológicas en las ciencias humanas y sociales de forma más abierta e integradora asumiendo otras perspectivas (fenomenológicas, hermenéuticas, dialécticas, críticas) como modelos de investigación con planteamientos claramente científicos que permiten la "búsqueda de significados sobre hechos particulares" que posibiliten obtener "argumentos para la acción", que es lo realmente importante.

No obstante, a pesar de esta tendencia hacia una epistemología plural, con frecuencia observamos que se habla, se opina, se indaga sobre los hechos y fenómenos educativos sin especificar previamente el marco epistemológico que se considera pertinente para que nuestra investigación posibilite la obtención de conocimientos científicos. Se actúa sin tener en cuenta que para identificar y establecer conocimientos científicos en el campo de la educación es necesario superar el plano apriorístico y empírico -basado en la especulación teórica, la observación asistemática y la experiencia no reglada- y avanzar en la reflexión sobre los fenómenos pedagógicos tratando de identificar sus regularidades -frecuencia, estructura, relación...- y las leyes por las que se rigen. En definitiva, como decía Piaget, (1975, p. 75) “traspasar los límites del empirismo superficial y con ayuda de la observación sistemática y el control poder leer la experiencia con lógica".

Por ello, cuando hablamos de pedagogía empírica, debemos asumir que "no todo vale" y que lo verdaderamente importante y necesario son los criterios de racionalidad científica que utilizamos en cada caso concreto para conocer y analizar los fenómenos y hechos educativos que nos permiten "pasar de las opiniones a las certezas". Tenemos la obligación de especificar las bases conceptuales teóricas y metodológicas que justifican el tipo de investigación por la que optamos en cada momento para "conocer" los fenómenos educativos ya que, de lo contrario, no se podría evitar la crítica ya formulada por Binet a comienzos del siglo pasado -“en pedagogía se ha dicho todo, pero nada se ha probado- y estaríamos negando el papel que ejerce la "ideología del investigador" a la hora de elegir una opción epistemológica. 


\section{Relevancia de problemas seleccionados}

La ideología del investigador no sólo se pone de manifiesto en su concepto sobre la naturaleza de la investigación empírica y el conocimiento científico sino también en la elección de los problemas que selecciona como objeto de estudio. Lo correcto sería que todos centremos nuestros trabajos sobre problemas relevantes pertinentes que respondan a las necesidades reales del sistema educativo que requieren una urgente respuesta. Sin embargo con frecuencia observamos que, siguiendo ideologías coyunturales y movimientos sociales puntuales, muchos investigadores focalizan sus trabajos sobre problemas que no son ni relevantes ni representativos de las necesidades reales del sistema -lo que en lenguaje coloquial se conoce como investigar sobre "el sexo de los ángeles"- dato que incide notablemente en nuestro descrédito ya que pone de manifiesto un acusado desconocimiento la realidad educativa.

A este respecto procede recordar que la finalidad primordial de todo trabajo de investigación empírica deberá ser la búsqueda de conocimientos nomopragmáticos -conocer/ decidir/mejorar- conocimientos cuyo valor depende de su utilidad para la toma de decisiones que permitan mejorar la práctica educativa. La calidad de los resultados de un trabajo de investigación educativa “dependerá tanto de su relevancia científica como de su oportunidad y adecuación para la toma de decisiones que contribuyan a la mejora" (De Miguel 1988, p. 66). Debemos tener claro que, si no hay mejora tangible, el trabajo resulta estéril. Y esto sí que es un problema. A la vista de los resultados, hasta la fecha nuestra aportación a la toma de decisiones para la mejora es bastante limitada y ello debe hacernos reflexionar a todos.

Si el objetivo final de toda investigación educativa es "conocer para mejorar la práctica" es lógico que constituya un requisito indispensable para quienes se dedican a investigar en este campo tener un conocimiento profundo de la realidad educativa sobre la que pretenden intervenir ya que, de lo contrario, muchos de los problemas planteados y las soluciones propuestas pueden ser consideradas como utópicas y descontextualizadas por quienes tienen que tomar las decisiones. Esto es lo que sucede como bastante frecuencia sobre todo en el ámbito universitario donde muchos investigadores se inician y realizan investigaciones sobre fenómenos educativos que no han experimentado personalmente y en contextos sociales que les son ajenos a su trayectoria vital. De alguna forma planifican trabajos y pretenden actuar sobre una realidad siendo ellos mismos "un extraño en el barrio" (Bok, 2008).

Por ello, procede recordar una vez más lo que ya decía Binet (1909, p. 72): "las cuestiones fundamentales de la educación y la pedagogía no se resuelven con teorías personalistas sino con el estudio lento, paciente y minucioso de los hechos en el mismo lugar que se producen". El mensaje es inequívoco. En la investigación educativa deben prevalecer como problemas a investigar las necesidades reales del sistema de cada contexto frente las ocurrencias generadas por las "teorías personalistas del investigador". Si el objetivo primordial que define la investigación educativa es mejorar la práctica, es lógico que se considere prioritario que los investigadores en este campo sean conocedores expertos sobre esta realidad que pretenden investigar y agentes comprometidos en la mejora de la misma. 
Este es un requisito insoslayable que pocas veces se cumple, como fácilmente se puede comprobar a través de los trabajos que se publican en revistas especializadas -como la presente- ya que en la selección de los problemas prevalecen los intereses y creencias peculiares del investigador a las necesidades del sistema educativo. Una vez más procede constatar que la falta de criterio y realismo del investigador contamina los objetivos y resultados de su trabajo haciendo que sus conclusiones sean muy poco útiles y que pueda ser tipificada su tarea por los prácticos como la de un extraño que vive aislado en su "torre de marfil". La elección de los problemas que son objeto de estudio constituye una prueba inequívoca del conocimiento y cercanía que el investigador manifiesta sobre las necesidades más urgentes del sistema educativo que necesitan ser remediadas (Bok, 2008).

\section{El paradigma de investigación como un escudo defensivo}

Los supuestos ideológicos del investigador no sólo condicionan la selección de los problemas a investigar sino también de la elección del marco metodológico que se considera pertinente para llevar a cabo en cada caso el proceso investigador. Lo normal debería ser elegir el paradigma de investigación en función de la naturaleza del fenómeno y la finalidad que se pretende alcanzar. Ambas cuestiones son fundamentales a la hora de optar por un determinado paradigma o enfoque metodológico para llevar a cabo el proceso investigador. Sin embargo, con demasiada frecuencia, se recurre -desde una posición ideológica predeterminada-a elegir "a priori" una opción paradigmática buscando en sus postulados argumentos teóricos y metodológicos de los que -en muchos casos- carece el propio investigador.

Nadie discute que el concepto de paradigma puede entenderse como un conjunto de supuestos epistemológicos que dirigen el proceso investigador hacia la búsqueda de nuevos conocimientos. Y que estos "supuestos epistemológicos" suelen ser comunes en el grupo de personas -comunidad científica-que realizan un determinado tipo de investigación. Ahora bien, no pertenecemos a una comunidad científica por el simple hecho de que nos ocupamos de investigar sobre problemas relativos a un determinado campo del conocimiento sino porque "compartimos un conjunto de afirmaciones teóricas y conceptuales sobre la naturaleza de los fenómenos objeto de estudio y los procedimientos adecuados para su investigación (De Miguel, 1988, p. 66). Y estos siempre deben estar explícitos y justificados.

El recurso a optar por un determinado paradigma o enfoque metodológico sin una discusión previa sobre su adecuación a la naturaleza del fenómeno a investigar es un hecho muy habitual en la investigación educativa que queremos poner de manifiesto de forma crítica. La mayoría de las investigaciones realizadas se limitan a señalar que utilizan un enfoque o paradigma metodológico cuantitativo, cualitativo o mixto basándose en que se utilizan una técnicas concretas para la recogida de información y con esta simple constatación eluden la necesaria discusión previa de carácter epistemológica sobre la naturaleza del objeto a investigar y el método que procede utilizar en cada caso. Más aún, la tendencia muy común a optar por una metodología mixta cuantitativa-cualitativa más que una coherencia teórica denota un recurso del investigador incapaz de justificar su opción dado que es difícil mantener un equilibrio y relación adecuada entre ambos enfoques metodo- 
lógicos. Se utiliza esta alternativa como un "escudo defensivo" que no compromete al investigador y que le permite justificar todo tipo de procesos y estrategias técnicas.

Si queremos considerar la pedagogía como una disciplina científica, al igual que en el resto de las ciencias, necesitamos una constante reflexión sobre la lógica de la investigación a partir de los desarrollos conceptuales en este campo y de la práctica de investigación educativa. Y es aquí, desde nuestro punto de vista, donde nuestra tarea vuelve a estar condicionada por la ideología del investigador sujeta a modas y filosofías coyunturales (Dendaluce, 1999). La tendencia habitual a situar dentro de un paradigma, modelo o enfoque determinado las investigaciones que se realizan porque se utilizan unas técnicas específicas de recogida de datos denota una carencia importante ya que lo procedente es que las decisiones metodológicas deben ser tomadas como una consecuencia de la opción epistemológica elegida que se justifica como más idónea para "conocer" un objeto o fenómeno concreto en un contexto social específico.

\section{Metodología científica y construcción del conocimiento}

Además del enfoque o paradigma elegido para llevar a cabo una investigación educativa es muy importante que el método que se utilice para la construcción del conocimiento sea científico; es decir, se fundamente sobre una lógica probatoria que permita la identificación de "evidencias". El conocimiento empírico solo adquiere el rango de científico cuando se fundamenta sobre "pruebas". Con frecuencia se tiende a considerar que el "argumentario probatorio" de la investigación educativa es débil $\mathrm{y}$, por tanto, carente de la solidez necesaria, sobre todo si se compara con el que se utiliza en el ámbito de las ciencias naturales. De ahí que, frecuentemente, se cuestionen los resultados de los trabajos al no estar justificados desde el punto de vista científico con una lógica concluyente (Henningsen, 1984).

La preocupación por utilizar metodologías basadas en "argumentos concluyentes" siempre ha estado presente entre los pedagogos empíricos. De hecho, durante las últimas décadas, se han ido adoptando distintos patrones o marcos de referencia metodológicos para la construcción y verificación de las hipótesis en función de la prevalencia ideológica de una determinada corriente o teoría epistemológica en el ámbito de las ciencias humanas y sociales. Por ello cabe establecer cierto paralelismo entre el tipo de epistemología dominante en cada momento en la investigación social y el procedimiento metodológico que se establece como patrón o modelo para la investigación educativa. A título de recordatorio, apuntamos brevemente los principales modelos utilizados durante las últimas décadas tratando de aportar una lógica científica al quehacer del investigador en el campo educativo.

\section{La pedagogía experimental como propuesta}

Probablemente muchos de los lectores desconozcan que, hasta la promulgación del Real Decreto 1888/1984 de 26 de septiembre que estructuró los distintos campos del saber en Áreas de Conocimiento, la disciplina académica que se ocupaba de la metodología de la investigación educativa se denominaba Pedagogía Experimental. La utilización de esta denominación disciplinar lógicamente da cuenta del enfoque 
tradicional que se venía asumiendo como marco de referencia para la investigación pedagógica: la búsqueda de conocimiento sobre los fenómenos educativos utilizando el método hipotético-deductivo-experimental. Las notas que caracterizaban esta disciplina según las definiciones de los autores clásicos que a comienzos del siglo XX se han ocupado de ella (Simon 1924, Claparéde 1931, Buyse, 1935) son las siguientes: a) estudio de los hechos pedagógicos b) de carácter empírico c) utilizando el método científico propuesto por Claude Bernard (1868) y d) con la finalidad de perfeccionar los procedimientos y métodos de enseñanza y educación. En definitiva, se definía su campo disciplinar en función de una metodología centrada sobre el experimento.

Lógicamente el término "pedagogía experimental" era la traducción al terreno pedagógico del espíritu científico positivista de una época, en estricta correspondencia con el de psicología experimental. La pedagogía, en la medida que quería ser una ciencia, no podía quedar al margen de este movimiento innovador que suponía la introducción de la metodología experimental propuesta por Claude Bernad (1868) en el ámbito de las ciencias humanas y sociales. De ahí que durante muchos años existiera cierta preocupación por dotar a esta disciplina de un estatuto epistemológico construido a partir del experimento a pesar de que los diseños metodológicos efectuados en este campo contaban con bastantes dificultades y problemas. Lógicamente, este enfoque restrictivo de la investigación educativa no sólo ha generado la crítica de otros colegas sino también que muchas de las metodologías alternativas al experimento hayan tenido un desarrollo tardío o se hayan impulsado desde otros sectores académicos ajenos a esta disciplina. Afortunadamente hoy se asume con normalidad que, ante imposibilidad de controlar todas las variables que condicionan los fenómenos educativos, el método experimental no es necesariamente el más idóneo para ser utilizado en la investigación educativa.

\section{La generalización empírica como criterio de racionalidad}

Dadas las limitaciones de la metodología experimental en el ámbito de la educación, los investigadores tratan de ampliar su campo de acción y fundamentar científicamente su trabajo utilizando procesos metodológicos que impliquen un contrate empírico con la realidad. La denominación empírico-experimental, en vez de solamente experimental, posibilitaba un enfoque más amplio sobre el concepto de la pedagogía como disciplina científica ya que permitía considerar e incluir la observación sistemática como método científico de investigación sobre los fenómenos educativos. Considerar la observación científica como otro método de investigación científica en el campo educativo implicaba que a través de esta metodología se podrían "descubrir leyes o generalizaciones sobre los fenómenos pedagógicos que pueden ser utilizadas para formular predicciones y controlar eventos dentro de situaciones educacionales" (Traves, 1979, p. 20) ya que, de lo contrario, se podría cuestionar el estatuto epistemológico de la pedagogía como ciencia. Por ello no era suficiente plantear la observación como método de investigación sino que era necesario precisar que ésta debería, al menos, posibilitar la "obtención de generalizaciones sobre las supuestas relaciones entre dos o más variables que inciden sobre los fenómenos educativos" (Traves, 1979, p. 26).

El concepto de "metodología empírico controlada" -término acuñado por Nagel (1981) - se considera en este momento como un nuevo patrón metodológico de la inves- 
tigación educativa en la medida que posibilita obtener generalizaciones a partir de las observaciones empíricas sin utilizar la manipulación de los fenómenos como se requiere en el experimento. El hecho de que el procedimiento habitual para poder establecer "generalizaciones empíricas" sea utilizando criterios estadísticos o probabilísticos ha generado una polarización de los investigadores sobre los registros numéricos de los datos, condicionando tanto el tipo de fenómenos a observar como las variables objeto de observación. De ahí que de la lógica del experimento hayamos pasado a la lógica del modelo estadístico dado que, bajo la presunción de la normalidad de los fenómenos y la homogeneidad de las unidades de análisis, permite realizar inducciones probabilísticas y, en consecuencia, establecer posibles relaciones funcionales entre variables de los fenómenos observados.

\section{La utilización de modelos matemáticos}

Dadas las limitaciones de las "generalizaciones empíricas" para establecer relaciones causales entre fenómenos, durante las últimas décadas los investigadores se han esforzado en la construcción y verificación de modelos que permitieran avanzar posibles relaciones causa/efecto entre diversas variables de los fenómenos analizados. Para ello han trasladado, mediante un proceso de formalización y modelización de las previsibles relaciones entre las variables, las hipótesis propuestas en los trabajos a modelos matemáticos susceptibles de ser comprobados mediante complejos métodos estadísticos. Así se han generado múltiples investigaciones educativas cuantitativas con diseños avanzados (causales, log-linear, jerárquico lineales, etc.) apoyándose en el posible isomorfismo existente entre teorías empíricas sobre las relaciones entre los fenómenos educativos y estructura de determinados modelos matemáticos que pueden ser verificados mediante métodos estadísticos, sobre todo cuando se dispone de su implementación informática.

El principal problema que cuestiona la utilización de estos "modelos causales" en la investigación social es la adecuación entre la estructura lógica subyacente en teoría utilizada para explicar las relaciones entre los fenómenos y las propiedades del modelo de análisis empírico. Frecuentemente sucede que la relación isomórfica entre teoría y modelo no siempre se establece adecuadamente ya que suele predominar lo que se conoce como "teoría del dato" al plantear las hipótesis preferentemente a partir de los análisis implícitos en cada "paquete estadístico" para que sea fácil su verificación sin atender a las relaciones lógicas subyacentes entre las variables de los fenómenos observados. Ello ha generado un predominio del dato estadístico descontextualizado sobre la teoría que se somete a comprobación lo que no ha contribuido positivamente a la valoración de esta tipo de metodología como adecuada para de llevar a cabo investigaciones sobre fenómenos de naturaleza interactiva que son los habituales en la realidad educativa.

\section{Comprensión frente a explicación. Las claves de un dilema}

La orientación claramente cuantitativa adoptada en la mayor parte de los trabajos realizados en la década de los setenta ha generado dialécticamente una actualización 
del dilema clásico sobre la finalidad de la investigación educativa entre explicación y comprensión. Tal como señalaba Wright (1979), ya hemos apuntado que la tradición científica de orientación aristotélica se fundamenta sobre explicaciones dadas en términos de finalidades, de intenciones, de motivos, de razones que permiten comprender los fenómenos y hechos educativos (hermenéutica). La tradición galileana identifica la explicación científica mediante la relación causal entre las variables controladas sobre los fenómenos dejando poco espacio a las finalidades. Constituyen, por tanto, dos formas de abordar el trabajo investigador que alimentan el clásico debate -no resuelto de modo satisfactorio- entre explicación y comprensión.

En la década de los ochenta, en buena parte alimentada entre nosotros por la publicación del texto de Cook y Reichardt (1982), Métodos cualitativos y cualitativos en la investigación educativa, la polémica en torno a la idoneidad y posibilidades que ofrece la metodología cualitativa en el estudio de los fenómenos educativos generó a su vez que aumentaran las críticas sobre el trabajo de quienes hasta el momento se habían ocupado en configurar la investigación educativa como una disciplina metodológica centrada sobre un enfoque empírico estadístico y probabilístico. De alguna forma, lo moderno, lo "progre", era apostar por una metodología cualitativa -basada más en la clasificación que en la cuantificación- como más adecuada para investigar los fenómenos educativos. Influidos por esta corriente comienzan a realizarse muchas investigaciones cualitativas aunque, en sentido estricto, muchas de ellas no pueden ser consideradas como científicas debido a su falta de rigor metodológico y otras no podrían ser consideradas estrictamente cualitativas pues se trata más bien de análisis cuantitativos de datos textuales: Atlas.ti, NVIVO, ...

No cabe duda que la publicación del texto de Miles y Huberman (1984) - Qualitative data análisis. A sourcebook of new methods y, sobre todo, el de Lincoln y Guba (1985), Naturalistic Inquir, constituyeron una aportación metodológica importante y novedosa para dotar de rigor al proceso y validez a los resultados en toda investigación fundamentada en un paradigma cualitativo e interpretativo. La no traducción de estos textos al castellano ha dificultado la comprensión y utilización correcta de las exigencias procedimentales necesarias cuando se utiliza esta metodología, y de los criterios lógicos que permiten juzgar el rigor científico de un proceso investigador teniendo en cuenta su validez interna, validez externa, fiabilidad y objetividad. La utilización de estos criterios, y de los estándares establecidos al respecto, no siempre constituye un objetivo prioritario de los investigadores que optan por este tipo de metodología, lo que debilita la fundamentación científica de su trabajo y determina que muchos de los resultados que aportan no sean transferibles.

\section{El pluralismo integrador como estrategia metodológica}

Una vez superados los enfrentamientos iniciales, a medida que se constatan las posibilidades y limitaciones tanto de los enfoques cuantitativos como de los cualitativos, surge una pregunta inevitable; ¿cúal de ellos es más idóneo para investigar los fenómenos educativos? La respuesta no era fácil. En el campo de la educación el dilema que ya planteaba Claparède (1931, p. 92) es muy aclaratorio al respecto. "Ante la pregunta hecha a un amigo ¿Por qué corres? caben dos respuestas: a) determinados 
procesos psíco-químicos activan los centros motores de los que dependen los músculos de las piernas, b) para no perder el tren". Así pues, frente a la explicación causal está la explicación teleológica a la que podemos llegar mediante la comprensión, o, dicho de otro modo, no sólo cabe analizar las causas que explican una conducta sino también las razones que la motivan. Claparède no elige una vía y excluye otra, sino que considera que hay que tomarlas sucesiva o simultáneamente, pues ellas se complementan. Basados en esta afirmación o creencia son muchos los investigadores que han mantenido, y aún mantienen, la complementariedad entre ambos enfoques científicos (Morgan 1983, Bericat 1998).

La complementariedad, desde nuestro punto de vista, no ha constituido una buena solución al dilema planteado. Aunque desde el punto de vista operativo se empezaron a utilizar en las investigaciones educativas estrategias y técnicas metodológicas de manera complementaria, la discusión sobre los planteamientos teóricos de ambos tipos de investigaciones se fueron acentuando de manera exponencial dado que no habían sido resueltos. Ante una evidente situación de desconcierto y tensión, finalmente entre nosotros surge lo que se ha denominado "pluralismo integrador" (Dendaluce, 1998) que pretende primero reconocer la validez y profesionalidad de quienes trabajan con metodologías distintas y flexibilizar los posicionamientos exclusivistas para aceptar e incorporar otros procedimientos metodológicos en el modo de abordar los problemas y buscar soluciones. En palabras de este mismo autor, quienes defienden como marco de referencia un pluralismo integrador "no tratan de hacer combinaciones de dos metodologías, ni síntesis, ni sincretismos, cuyo producto sea algo nuevo distinto de las dos cosas de las que partimos. Lo que se propone es una mejora de la metodología que más nos convence en general o que nos parece más apropiada para el problema que estamos estudiando utilizando herramientas y procedimientos de la otra que nos puedan ser útiles" (Dendaluce 1998, p. 12).

La propuesta orientada hacia la búsqueda de un "pluralismo integrador" no ha tenido la respuesta esperada ya que, en vez de avanzar hacia una opción dialéctica donde se utilizaran las diferencias entre perspectivas metodológicas contrapuestas para avanzar en nuevos modos de conocimiento, se derivó hacia una actitud mas permisiva parecida a la epistemología radical de Paul Feyerabend (1981) en la que "todo vale". En vez de avanzar y mejorar mediante un proceso crítico que nos permitiera reflexionar sobre las bases epistemológicas que utilizamos como soporte de nuestras investigaciones optamos por una actitud ingenua sustentada sobre el principio de que no existe idea -por antigua o absurda que sea- que no contribuya a mejorar nuestro conocimiento. Ello ha generado un desprecio encubierto por la dimensión teórica que debe sustentar toda investigación y, en consecuencia, una falta de rigor y coherencia que observamos en muchos de los diseños realizados y que queremos denunciar explícitamente.

\section{El regreso a la experiencia como marco de referencia}

El rasgo más característico de la etapa más reciente de la investigación educativa es la sobrevaloración de la experiencia como marco teórico de referencia para el estudio de los fenómenos educativos. No importa tanto el enfoque epistemológico que se elige 
para abordar el estudio de los fenómenos educativos como el hecho de utilizar una metodología fundamentada sobre la experiencia personal; es decir, aquello que tiene "significado" para el individuo (Morin, 1984). Ello nos remite a un concepto de "experiencia" como "conjunto de conocimientos, especialmente de índole práctico, que se adquieren en la vida diaria o en el ejercicio de una ocupación". En consecuencia, el término conocimiento empírico alude a un tipo de conocimiento práctico obtenido desde la experiencia; es decir, que toma la experiencia como referencia básica del conocimiento humano. Ello justifica la preocupación de los investigadores por codificar todas las observaciones tomadas en la práctica diaria en datos objetivos y manejables a partir de los cuales se puedan efectuar alguna tentativa científica al nivel más elemental (Mouly, 1978).

Esta necesidad de contar con datos empíricos manejables ha constituido y constituye el objetivo prioritario de la mayoría de las investigaciones empíricas ya que permiten -dentro de unos márgenes de error- extraer algún tipo de conclusiones aunque no puedan ser generalizables. Por ello procede señalar que la utilización de datos empíricos en la investigación educativa conlleva una referencia a cierto tipo de conocimientos prácticos pero no significa que sean claramente científicos. Una cosa es que el conocimiento sea empírico y otra distinta que además sea científico. De nuevo hemos caído en un error que es necesario aclarar: ningún dato empírico puede ser utilizado como válido sin tener en cuenta la teoría que sustenta el procedimiento metodológico que ha permitido su obtención. Lo que de nuevo nos vuelve a plantear la necesidad de reflexionar sobre la consabida interdependencia entre la teoría y el dato. El recurso a la "experiencia personal" no resuelve esta mutua interdependencia (Stegmuller, 1979).

\section{A modo de síntesis: entre Scilla y Caribdis}

A la vista de lo señalado en los apartados anteriores, podemos concluir que a lo largo de las últimas décadas se han utilizado diversos "argumentarios lógicos" como criterios de racionalidad científica para avalar los "conocimientos" obtenidos en los trabajos de pedagogía empírica. Los investigadores siempre han gozado de plena libertad para realizar sus trabajos tanto a la hora de elegir el problema objeto de la investigación como la metodología que han considerado oportuna para llevarlo a cabo. Nadie les impone sobre qué deben investigar y cómo hacerlo. Por ello, las elecciones y decisiones que se toman en cada caso concreto deben entenderse mediatizadas por su opción ideológica sobre qué consideran como conocimiento científico en el ámbito de la educación y, sobre todo, cómo se construye este tipo de conocimiento.

A título de síntesis cabe decir que durante los últimos años los investigadores empíricos en educación hemos navegado entre Scilla y Caribdis -entre metodologías que buscan la explicación causal de los fenómenos y las que interpretan la experiencia personal aunque sea poco transferible- pero la mayoría de las veces en los trabajos no se aportan las coordenadas geográficas concretas -las claves metodológicas- por lo que resulta difícil su ubicación teórica y corren el riesgo de "estrellarse". Dado que existe una pluralidad de procedimientos lógicos probatorios, cada uno con sus propias limitaciones, cuando el investigador no justifica de forma científica y correcta la opción metodológica que elige da pie a que pueda ser juzgado más por su "ideología" que por la "lógica" que utiliza para la construcción del conocimiento. 


\section{Difusión interesada los trabajos realizados}

Ya hemos comentado que el reto que debe orientar todo trabajo de investigación empírica en el campo de la educación es mejorar la práctica educativa. Más allá de la libertad e independencia inherente a todo quehacer científico, el investigador debe tener presente la responsabilidad individual que contrae con la sociedad por lo que debe estar muy atento a los beneficios sociales que se derivan de los resultados obtenidos. De ahí que debamos tener como norma no sólo que nuestro trabajo constituya una aportación al conocimiento sino que además tenga aplicaciones prácticas evidentes, especialmente cuando sus proyectos están financiados con fondos públicos.

Sin embargo, una de las críticas más generalizadas que se formulan sobre la investigación educativa es su escasa aportación a la mejora de la educación práctica. La reacción corporativa más habitual es justificar la escasa utilidad de nuestros trabajos utilizando factores ajenos al propio investigador. Los argumentos que se utilizan para contrarrestar esta crítica son muy diversos y casi siempre exculpatorios al poner énfasis en factores ajenos a la propia investigación -dadas las condiciones y limitaciones en las que se llevan a cabo los proyectos (escasez de programas y fondos, dotaciones para formar investigadores, asignación de recursos, etc.) - y a que los resultados no se aprovechan ante falta de interés de quienes tienen que tomar las decisiones pertinentes.

Si tratamos de ver la "otra cara de la moneda" y planteamos como perspectiva de análisis lo que el investigador debe hacer para que sus trabajos tengan utilidad esperada la cuestión es distinta. Desde un punto de vista práctico consideramos que existen tres factores que nos parecen determinantes cara a la utilización de las conclusiones de una investigación: la clarificación de los resultados y propuestas de mejora que se derivan de cada trabajo, la especificación de las decisiones a tomar y quién debe tomarlas, y la difusión de la información pertinente entre las audiencias implicadas en cada proyecto realizado. No tener en cuenta estas tareas nos llevaría a pensar que la finalidad principal del investigador no se orienta hacia el objetivo que debe regir en toda investigación educativa: construir conocimiento que permita mejorar la práctica.

Sin embargo, todos somos conscientes que la realidad es otra ya que el objetivo fundamental para la mayoría de los investigadores es publicar sus trabajos en revistas con elevado índice de impacto con el fin de que puedan reportarle rentabilidad en su carrera académica. Ello le lleva adaptar la difusión de sus trabajos a las reglas que imponen las revistas científicas y a configurar la publicación siguiendo las normas establecidas al respecto (antecedentes, estructura, cita, etc.) con el fin de superar el proceso previo de evaluación, al margen de lo que sería oportuno para que llegue a quienes tienen que tomar las decisiones pertinentes. Así, se dan casos extremos y paradójicos en los que algunos investigadores centran todos sus esfuerzos sobre el soporte metodológico o publican en revistas extranjeras especializadas trabajos en los que la principal audiencia implicada son los profesores de educación infantil y primaria.

Todo ello pone de relieve que se antepone el interés personal del investigador a lo que sería deseable desde la perspectiva de la utilización de los resultados. Frente a la lógica propia de la investigación educativa -conocer, decidir, mejorar- se impone otra secuencia mediatizada por una decisión personal del investigador donde lo verdaderamente importante es lograr situar sus trabajos en las revistas con mayor prestigio al 
margen de los impactos que puedan tener. Esta acomodación a las reglas del mercado -recabar fondos/publicar/acreditar- no solo pone de relieve la finalidad primordial que motiva al investigador sino que desvela igualmente una acusada carencia del compromiso ético que debe regir toda actividad científica.

\section{Utilización de códigos éticos}

A lo largo de los apartados anteriores hemos puesto de relieve algunos de los problemas y dilemas que se plantean a lo largo del proceso investigador y que habitualmente se resuelven utilizando más una posición u opción ideológica que lógica. Todos somos conscientes de que esto es un hecho bastante común a toda actividad humana por lo que sería impensable que la actividad del investigador en el ámbito educativo pudiera permanecer al margen. Ahora bien, una cosa es reconocerlo y otra distinta no hacer nada para controlar este condicionamiento ideológico subyacente que adultera y contamina todo el proceso investigador y la utilidad de los resultados.

Por ello, nuestra llamada de atención sobre la responsabilidad social de los investigadores no se limita a enfatizar la necesidad de que los resultados obtenidos con sus trabajos sirvan para mejorar las prácticas educativas sino también a la exigencia de actuar con rigor y sentido ético en todas las decisiones tomadas a la largo del proceso realizado para la obtención de las evidencias. De ahí que adquiera pleno sentido la utilización de un código ético o deontológico como expresión de las normas a tener en cuenta por el investigador ante las diversas cuestiones y dilemas que se le plantean en sus actuaciones profesionales y que transcienden a la opción paradigmática elegida ya que esta elección -como ya hemos señalado- también está en buena medida mediatizada por su ideología.

Ahora bien, remitir a la exigencia de que el investigador suscriba un código ético o deontológico no resuelve definitivamente el problema del condicionamiento que la ideología del investigador genera sobre el proceso y utilidad de los resultados ya que la mayoría de estos códigos se construyen sobre las "competencias" que se consideran necesarias para ejercer la función investigadora o sobre los "estándares" de calidad que deben orientar sus actuaciones profesionales, pero no suelen indicar la decisión que se debe tomar en cada caso concreto cuando se ponen en juego opciones teóricas y metodológicas contrapuestas. Más bien se orientan a lograr que el investigador sea competente profesionalmente y que su trabajo pueda ser tipificado como "buenas prácticas", tal como se puede verificar en el código publicado por la AERA (1992) elaborado a partir de cuarenta y seis estándares organizados en seis apartados que incluyen a todos los sujetos implicados en la gestión de los procesos de creación y difusión del conocimiento (investigadores, administradores, editores, patrocinadores, etc.).

Por ello, coincidimos con Tojar y Serrano (2000) que la utilización más adecuada de los códigos deontológicos es como materiales de reflexión y debate que permiten a los investigadores revisar lo que hacen de forma crítica. De ahí que consideramos necesario que en toda investigación haya un apartado final destinado a que el investigador explique los dilemas que le ha planteado su trabajo y exponga las razones que le han llevado en cada caso a elegir una determinada opción. Explicar estas cuestio- 
nes nucleares en todo proceso de investigación y los dilemas de tipo ideológico que conllevan es una exigencia ética insoslayable si queremos que las opciones elegidas en nuestro trabajo estén justificadas. Este es el objetivo prioritario que ha impulsado la redacción de este texto: efectuar una llamada de atención sobre la necesidad de llevar a cabo una reflexión ética sobre la ideología que subyace en nuestros trabajos como investigadores empíricos en el ámbito educativo con la finalidad de identificar algunas debilidades que podamos superar.

\section{Cuestiones para un debate}

Situados en este contexto, vamos a formular algunas cuestiones que pueden ser objeto de esta reflexión que consideramos necesaria y que hacemos asumiendo como nuestros algunos de los interrogantes que la doctora Bartolomé (2000) formula en su excelente trabajo Hacia donde va la investigación educativa. Con el fin de que nuestra aportación pueda entenderse como una especie de guía para la reflexión, y el consiguiente debate, estructuramos la propuesta en seis apartados correlativos a los puntos fundamentales abordados en este artículo; a saber:

a. Recuperar el debate epistemológico

Las cuestiones epistemológicas no están de actualidad entre los investigadores empíricos que se dedican al campo educativo. Basta analizar el número de trabajos que se publican en las revistas especializadas para darse cuenta del escaso interés que despiertan este tipo de problemas sobre todo si lo comparamos con lo que sucedía en las décadas de los setenta y los ochenta (Dendaluce, 1999), Por ello entendemos como necesario abrir un debate en torno a algunas cuestiones como: ¿Qué entendemos por investigación científica en el campo de las ciencias sociales y humanas?, ¿Contamos en el campo educativo con un corpus de conocimientos científicos adquiridos mediante investigaciones rigurosas? ¿Cuáles son las principales dificultades que impiden considerar el trabajo de los investigadores en el campo educativo como realmente científico?, ¿Qué papel ejercemos los pedagogos empíricos dentro del marco de las Ciencias de la Educación?, ¿Qué aportamos?,¿Cuáles son nuestras fortaleza y nuestras debilidades?, etc.

b. Focalizar los proyectos sobre problemas relevantes y urgentes.

Buena parte de las críticas que se formulan a la investigación educativa tienen que ver con su ineficacia para solucionar los problemas reales. Partiendo de este supuesto, las preguntas son inevitables: ¿Nuestros trabajos aportan conocimientos científicos concluyentes relacionados con las necesidades del sistema?, ¿Hemos contribuido de manera significativa al progreso del conocimiento en el ámbito educativo?, ¿Por qué se están haciendo tantas críticas a la investigación educativa?, ¿Tiene algo que ver con su capacidad de respuesta a los problemas sociales?, ¿Aportamos certezas que pueden ser tenidas en cuenta para la toma de decisiones?, ¿Hasta qué punto la elección de los temas y problemas a investigar está condicionada a la ideología personal del investigador y no por las necesidades del sistema?, etc. 
c. Justificar el paradigma y enfoque metodológico utilizado

El debate metodológico actual no gira tanto sobre cuál de los tres paradigmas clásicos es más pertinente en la investigación social cómo sobre la necesidad de reflexionar acerca de la utilización que se hace de los supuestos teóricos y planteamientos metodológicos de estos paradigmas en cada trabajo científico. No se trata, por tanto, de cuestionar cuál de estos paradigmas es más pertinente en el campo educativo sino de analizar si se hace un "adecuado uso" del enfoque elegido en cada investigación concreta. Por ello necesitamos argumentar adecuadamente nuestras decisiones al respecto reflexionando sobre cuestiones como: ¿Cuáles son los supuestos teóricos que justifican la elección del paradigma utilizado?, ¿Qué argumentos avalan esta elección?, ¿Qué implicaciones conlleva desde un punto de vista metodológico?, ¿Qué tipo de procedimientos y técnicas son coherentes con el enfoque metodológico elegido?, etc.

d. Especificar el tipo de soporte científico que avala las evidencias obtenidas El criterio determinante para identificar un conocimiento como científico son las evidencias obtenidas mediante el proceso de investigación. Ahora bien, estas evidencias puedan ser obtenidas por distintos procedimientos no siempre rigurosos y contrastables. Las cuestiones a debatir surgen de forma inmediata: ¿Se ha elegido un procedimiento metodológico adecuado a la naturaleza del problema planteado?, ¿Se ha utilizado de forma correcta y parsimoniosa el método elegido para la obtención de las evidencias?, ¿Se han establecido los adecuados sistemas de control de los datos y los análisis realizados?, ¿Se ha efectuado una revisión crítica de los resultados obtenidos?, ¿Se ha procedido al contraste de las evidencias con otros estudios sobre la misma temática?, ¿Existen trabajos de síntesis y metaanálisis sobre este temática?, etc.

e. Reorientar los procesos de difusión y diseminación.

Ya hemos comentado que la difusión de los trabajos científicos no puede quedar sólo limitada a las publicaciones especializadas. Es necesario promover la comunicación e intercambio entre investigadores e instituciones superando las diferencias culturales y lingüísticas, y sobre todo con las audiencias implicadas en la toma decisiones que posibiliten la mejora. De ahí que debamos reflexionar sobre cuestiones como: ¿Cuáles han sido los propósitos fundamentales que han orientado nuestros trabajos?, ¿Qué utilidad han tenido los resultados obtenidos en la transformación de la práctica educativa?, ¿Cómo elaborar y difundir los informes para que lleguen a las audiencias que deben tomar decisiones?, ¿Qué uso efectuamos de las redes sociales para diseminar los resultados y promover la utilización de los datos?, etc.

f. Revisar nuestro compromiso ético

Finalmente hemos señalado que el control de la ideología en la investigación empírica es ante todo un problema ético que sólo tiene una respuesta adecuada desde el compromiso personal del investigador con unos valores que van más allá de sus competencias profesionales y el cumplimiento de los estándares 
de calidad establecidos al respecto. Por ello entendemos que en este ámbito la reflexión siempre debe estar abierta de forma permanente a preguntas como:¿Qué actitudes éticas debemos adoptar en la investigación educativa?, ¿Cuál es nuestro grado de cumplimiento de los códigos y normas establecidos al respecto?,¿Cómo aseguramos su calidad y el rigor de nuestras actuaciones profesionales?, ¿Qué valoración crítica efectuamos sobre las aportaciones de nuestro trabajo?, ¿Qué procedimientos utilizamos para controlar que nuestra ideología e intereses personales no prevalezcan frente a las necesidades sociales?, etc.

Estas y otras preguntas similares son las que quisiéramos sugerir con este trabajo no tanto para dar soluciones -tarea realmente difícil- como para promover la reflexión y el debate entre todos que consideramos necesario. Los investigadores en educación gozan de plena libertad para realizar sus trabajos, elegir el problema a investigar, utilizar la metodología que consideran oportuna, aportar su interpretación personal de los resultados y decidir cómo, dónde y cuándo comunicar y difundir sus trabajos. Nadie les dice qué investigar y cómo hacerlo, pero es necesario que se cuestionen si lo que hacen cumple las exigencias y el compromiso ético que nuestra sociedad reclama a toda persona que emplea recursos a favor del bien común.

\section{Referencias}

American Educational Research Association (1992). Ethical Standards of the American Educational Research Association. Washington: A.E.R.A.

Bartolomé, M. (2000). Hacia donde va la investigación educativa. Barcelona: DULAC.

Bericat, E. (1998). La integración de los métodos cuantitativo y cualitativo en la investigación social. Barcelona: Ariel.

Binet, A. (1909). Les idées modernes sur les enfants. Paris: Flamnarion.

Bok. D. (2008). Más allá de la torre de marfil. Buenos Aires: Fundación Palermo.

Buyse, R. (1935). L'experimentation en pédagogie. Bruxelas: Lamertin.

Buyse, R. (1949). Origen y desarrollo de la pedagogía experimental. Rev. Española de Pedagogía, 28.

Castañé.J. (1986). Ideología pedagógica y realidad educativa. Rev. Teoría de la Educación, 1, 83-100.

Claparède, E. (1931). L'éducation fonctionnelle. Suiza: Delachaux y Niestle.

Cook, T. y Reichardt, (1982). Qualitative and quantitative methods in evaluation research. London: Sage.

De Miguel, M, (1988). Paradigmas de la investigación educativa. En Dendaluce, I. (Coord.) Aspectos metodológicos de la investigación educativa. Madrid: Narcea.

Dendaluce, I. (1995). Avances en los métodos de investigación educativa en la intervención psicopedagógica. Revista de Investigación Educativa, 26, 9-32.

Dendaluce, I. (1998). Algunos retos metodológicos. Revista de Investigación Educativa, 16(1), 7-24.

Dendaluce, I. (1999). La investigación educativa ente el tercer milenium, Rev. Bordón, 51(4), 363-376.

Feyerabend, P.K. (1981). Tratado contra el método. Madrid, Tecnos. 
Feyerabend, P.K. (1982). La ciencia en una sociedad libre. Madrid: Siglo XXI.

Henningsen, J. (1984). Teoría y métodos en la ciencia de la educación. Barcelona: Herder

Lakatos, I.et al. (1982). Historia de la ciencia y de sus reconstrucciones racionales. Madrid: Tecnos.

Lenk, K. (1974). El concepto de ideología. Buenos Aires: Amorrortu.

Mardones, J.M. (1991). Filosofa de las ciencias humanas y sociales. Barcelona: Anthropos.

Lincoln, Y. y Guba, E, y (1985). Naturalistic inquiry. Beverly Hills: Sage.

Miles, M. y Huberman, M. (1984). Qualitative data análisis. London: Sage.

Morgan, G. (ed.) (1983). Beyond method: Strategies for social research. Beverly Hills: Sage.

Mouly, G. (1978). Eduational research. The art and science of investigation. Boston: Allyn and Bacon.

Morin, E. (1977). El método. La naturaleza de la naturaleza. Madrid: Cátedra.

Morin, E. (1984). Ciencia con consciencia. Barcelona, Anthropos

Piaget, J. et al. (1975). Tendencias de la investigación en ciencias sociales. Madrid, Alianza.

Popkewitz, Th. (1988). Paradigmas e ideología en la investigación educativa. Las funciones sociales del intelectual. Madrid: Mondadori.

Rossi, F. (1980). Ideología. Barcelona, Labor.

Simon, Th. (1924). Pédagogie expérimentale. Paris: Colin.

Stegmuller, W. (1979). Teoría y experiencia. Barcelona: Ariel.

Tójar, J.C. y Serrano, J. (2000). Ética e investigación educativa. RELIEVE, 6(2).

Traves, R.M. (1979). Introducción a la investigación educacional. Buenos Aires: Paidós.

Trias,E. (1975). Teoría de las ideologías. Barcelona: Península.

Wright, C.H. (1979). Explicación y comprensión. Madrid: Alianza.

Fecha de recepción: 27/02/2015.

Fecha de revisión: 27/02/2015.

Fecha de aceptación: 01/05/2015. 
